\title{
Point-contact spectroscopy investigation of superconducting-gap anisotropy in the nickel borocarbide compound $\mathrm{LuNi}_{2} \mathrm{~B}_{2} \mathrm{C}$
}

\author{
N. L. Bobrov, ${ }^{1,2}$ S. I. Beloborod'ko, ${ }^{1}$ L. V. Tyutrina, ${ }^{1}$ I. K. Yanson, ${ }^{1, *}$ D. G. Naugle, ${ }^{2}$ and K. D. D. Rathnayaka ${ }^{2}$ \\ ${ }^{1}$ B. I. Verkin Institute for Low Temperature Physics and Engineering, NAS of Ukraine, 47, Lenin Prospect, 61103, Kharkiv, Ukraine \\ ${ }^{2}$ Department of Physics, Texas A\&M University, College Station, Texas 77840-4242, USA \\ (Received 22 April 2004; revised manuscript received 1 September 2004; published 18 January 2005)
}

\begin{abstract}
Point contacts are used to investigate the anisotropy of the superconducting energy gap in $\mathrm{LuNi}_{2} \mathrm{~B}_{2} \mathrm{C}$ in the $a b$ plane and along the $c$ axis. It is shown that the experimental curves should be described assuming that the superconducting gap is nonuniformly distributed over the Fermi surface. The largest and the smallest gaps have been estimated by two-gap fitting models. It is found that the largest contribution to the point-contact conductivity in the $c$ direction is made by a smaller gap and, in the $a b$ plane by a larger gap. The deviation from the one-gap BCS model is pronounced in the temperature dependence of the gap in both directions. The temperature range, where the deviation occurs, is for the $c$ direction approximately 1.5 times more than in the $a b$ plane. The $\Gamma$ parameter, allowing quantitative estimates of the gap anisotropy by one-gap fitting, in the $c$ direction is also about 1.5 times greater than in the $a b$ plane. Since it is impossible to describe satisfactorily such gap distribution either by the one- or two-gap models, a continuous, dual-maxima model of gap distribution over the Fermi surface should be used to describe superconductivity in this material.
\end{abstract}

DOI: 10.1103/PhysRevB.71.014512

PACS number(s): 63.20.Kr, 72.10.Di, 73.40.Jn

\section{INTRODUCTION}

The goal of this study was to investigate the anisotropy of the energy gap in nickel borocarbide superconductors $R e \mathrm{Ni}_{2} \mathrm{~B}_{2} \mathrm{C}$. The crystallographic structure of these compounds resembles to some extent the structure of HTSC materials. ${ }^{1}$ It consists of alternating $R e-\mathrm{C}$ and $\mathrm{Ni}_{2}-\mathrm{B}_{2}$ layers ( $R e$ is a rare earth metal). There is a point of view that the electron properties of $\operatorname{ReNi}_{2} \mathrm{~B}_{2} \mathrm{C}$ compounds in the normal state are isotropic because of the strong carbon bond along the tetragonal $c$ axis. ${ }^{2}$ This is supported by the temperature dependence of isotropical resistivity in the single crystal of $\mathrm{YNi}_{2} \mathrm{~B}_{2} \mathrm{C}{ }^{3}$ On the other hand, there is also data about a substantial anisotropy in these compounds. ${ }^{4}$ In the superconducting state the experimental data are also contradictory. The upper critical fields for $H$ parallel and perpendicular to the $c$ axis and the derived superconducting parameters do not show any anisotropy for the $\mathrm{YNi}_{2} \mathrm{~B}_{2} \mathrm{C}$ single-crystal samples in agreement with magnetization and torque magnetometry measurements, but a small anisotropy is observed for the $\mathrm{LuNi}_{2} \mathrm{~B}_{2} \mathrm{C}$ single crystals. ${ }^{3,5}$ Meanwhile, a very strong anisotropy of the superconducting energy gap was reported for $\mathrm{LuNi}_{2} \mathrm{~B}_{2} \mathrm{C}$ in the $a b$ plane. This conclusion was based on the observation of delocalized quasiparticles in thermal conductivity at very low temperatures. ${ }^{6}$ According to this analysis, the smallest gap differs at least 10 times from the gap in other directions. Recently, evidence for the presence of nodes along $\langle 100\rangle$ direction was provided by the field-angle thermal conductivity, ${ }^{7}$ field-angle heat capacity, ${ }^{8}$ and ultrasonic attenuation measurements of $\mathrm{YNi}_{2} \mathrm{~B}_{2} \mathrm{C}$. ${ }^{9}$

In accordance with the experimental data an anisotropic superconducting gap function was proposed in Ref. 10 [see, also, Fig. 52 from the recent review (Ref. 11)]. In that model the gap has nodes along $\langle 100\rangle$ directions and attains the maximum values along $\langle 110\rangle$ directions. In the case of $s+g$ symmetry, the elastic scattering leads both to a decrease of $T_{c}$ and to an "isotropization" of the gap with vanishing nodes.

We do not know any direct measurement of gap anisotropy involving the Andreev reflection. ${ }^{12}$ The STM tunnelling measurements at $4 \mathrm{~K}$ report a gap of $2.2 \mathrm{meV}$ along the $c$ axis, yielding a too low ratio $2 \Delta / k T_{c}=3.2$ for $\mathrm{LuNi}_{2} \mathrm{~B}_{2} \mathrm{C}$. ${ }^{13,14} \mathrm{By}$ contrast, there are point-contact measurements of the gap in the $a b$ plane (e.g., Refs. 15 and 16), which yield $2 \Delta / k T_{c}=3.7-3.8$. The point-contact spectroscopy method in the latter measurements cannot, however, provide angular resolution of the gap anisotropy much better than $\pi / 2$.

In the present investigation we have found that both in the $a b$ plane and in $c$ direction the experimental point-contact spectra cannot be fitted satisfactory with the one-gap theoretical curve, even when broadened by an adjustable broadening parameter. Fitting the experimental spectra both at a small bias $(e V<\Delta)$ and at a bias larger than $\Delta$ forces us to use at least the two-gap fitting curve like the one for the two-band superconductor. ${ }^{12,17}$ But even the two-gap fitting with the two proper broadening parameters was not good enough to approximate the experimental characteristic at the middle energy region $(e V \sim \Delta)$. Moreover, for the two-band model one could expect transitions of Cooper pairs between the bands to introduce an additional depairing factor, which is analogous to other depairing factors like magnetic impurities, strong magnetic fields, etc. In such case the superconducting order parameter and the energy gap should differ from each other, which could be accounted for by using Ref. 18. Unfortunately even using the latter theory satisfactory fits could not be obtained. Therefore we suggest that only a continuous gap distribution with the two energy gap maxima around the Fermi surface could satisfactorily approximate the experimental spectra at low temperature $\left(T \ll T_{c}\right)$. We have determined tentatively the value of these maxima and the range of gap distribution which corresponds well to the recent STM measurements. ${ }^{19}$ 


\section{EXPERIMENTAL TECHNIQUE}

The point contact measurement was performed on single crystal $\mathrm{LuNi}_{2} \mathrm{~B}_{2} \mathrm{C}$ with $T_{c} \simeq 16.9 \mathrm{~K}$ grown by Canfield and Bud'ko using a flux method. ${ }^{20}$ Geometrically, our crystal was a thin $(0.1-0.2 \mathrm{~mm})$ plate with the $c$ axis perpendicular to its plane. The single crystal surface always contains quite a thick layer in which superconductivity is either absent or strongly suppressed. To perform measurement in the $a b$ plane, the crystal is usually cleaved and the point contact is made between a metallic counterelectrode and the cleaved surface. It was technically problematic to produce a cleavage perpendicular to the $c$ direction. In this case the crystal surface was cleaned with a $10 \% \mathrm{HNO}_{3}$ solution in ethanol. As the measurement in the $a b$ plane shows, both cleavage and etching yield identical results.

The point contact in the $a b$ plane is fabricated between an edge of the silver electrode and a freshly cleaved (or etched) corresponding facet of the single crystal. ${ }^{21}$ The deviation from the perpendicular to the $c$ direction might amount to about $5^{\circ}-10^{\circ}$. We do not know a priori along which of the in-plane directions the contact is obtained. But since we used a selection rule to choose the highest observable superconducting energy gap with the largest nonlinearity at the gap double-minimum structure, the contact axis is presumably along the directions, where the in-plane gap is maximum. To produce contacts in the $c$ direction the traditional "needleanvil" geometry is used. The radius of the needle is about 1-3 micron. The temperature was measured using a special cryogenic insert (its close analogue is described elsewhere ${ }^{22}$ ).

The point contact resistances varied typically from several Ohms to tens of Ohms. For detailed investigations we chose the point contacts with the highest permissible tunneling which was controlled against the differential resistance maximum at zero bias and by the maximum nonlinearity in the $d V / d I$-double-minimum region, corresponding to the intact superconducting surface under the contact. Unfortunately, a complete set of curves in the whole $T_{\min }-T_{c}$ interval was obtained only for a few contacts. Because of their rather high resistance and the long-duration (over 10-12 hours) of the measurements, many of the contacts were broken down. The temperature interval ranges from the minimal available $T(\sim 1.5 \mathrm{~K})$ to $T_{c}$.

\section{RESULTS AND DISCUSSION}

Some curves of the first derivatives $d V / d I$ measured at different temperatures on $\mathrm{LuNi}_{2} \mathrm{~B}_{2} \mathrm{C}$-Ag point contacts along $c$ axis and in the $a b$ plane are shown in Figs. 1 and 2 .

The measured curves were symmetrized $d V / d I_{\text {sym }}$ $=1 / 2[d V / d I(V)+d V / d I(-V)]$ and normalized to the normal state at $T>T_{c}$, except in Figs. 1 and 2, where the raw data are shown. Each curve contains several hundred of experimental dots and at the scale shown in the presented figures, all the curves have negligible noise. In other figures experimental curves are shown by dots whose number is decreased in order to be discerned. Statistically (several tens of contacts were examined for each direction), the distance between the

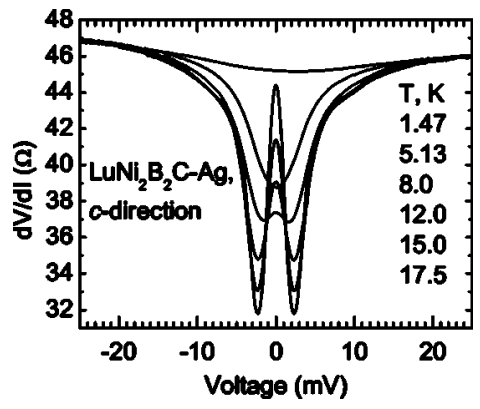

FIG. 1. Differential resistance of $\mathrm{LuNi}_{2} \mathrm{~B}_{2} \mathrm{C}-\mathrm{Ag}$ point contact at different temperatures. The contact axis is parallel to $c$. Not to overload the figure, we give only several representative curves.

minima in the first derivative $d V / d I$ characterizing the average value of the gap was $12-15 \%$ larger in the $a b$ plane compared to that in the $c$ direction. This is a very crude estimation of the $c \leftrightarrow a b$ anisotropy.

The theoretical predictions and the experimental results were compared using two approaches. First, a model was applied, which describes electrical conductivity of pure $S-c-N$ point contacts in the presence of an arbitrarily transparent potential barrier at the boundary between the metals. This model allows for the finite lifetime of the Cooper pairs. ${ }^{18}$ The $I-V$ characteristics of the point contact are described as follows:

$$
\begin{aligned}
I= & \frac{1}{2 e R_{N}} \int_{0}^{\infty} d \varepsilon\left[\tanh \frac{\varepsilon+e V}{2 T}-\tanh \frac{\varepsilon-e V}{2 T}\right] \\
& \times \frac{1}{\left|\left(2 d^{-1}-1\right)+g_{\varepsilon}^{R}\right|^{2}} \times\left\{\left|1+g_{\varepsilon}^{R}\right|^{2}+\left|f_{\varepsilon}^{R}\right|^{2}+4\left(d^{-1}-1\right) \operatorname{Re} g_{\varepsilon}^{R}\right\},
\end{aligned}
$$

where $g_{\varepsilon}^{R}=u / \sqrt{u^{2}-1}, f_{\varepsilon}^{R}=1 / \sqrt{u^{2}-1}, d=1 /\left(1+Z^{2}\right), d$ is the transparency of the potential barrier, and $Z$ is the tunneling parameter. The value $u=\varepsilon / \Delta_{\varepsilon}\left(\Delta_{\varepsilon}\right.$ is a complex energyindependent order parameter of the superconductor), which can be found from the following equation: ${ }^{23}$

$$
u=\frac{\varepsilon}{\Delta}+i \gamma \frac{u}{\sqrt{u^{2}-1}},
$$

where $\Delta$ is the BCS order parameter obtained from

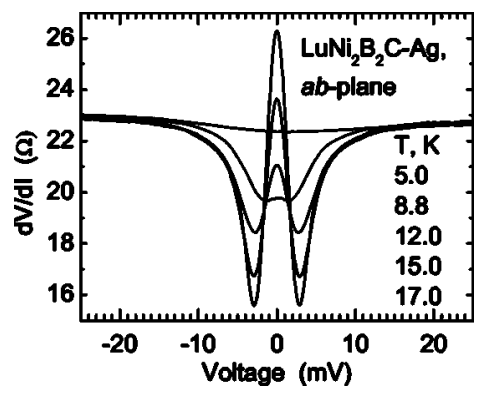

FIG. 2. Differential resistance of $\mathrm{LuNi}_{2} \mathrm{~B}$ C-Ag point contact at different temperatures. The contact axis is along the $a b$ plane. See the last sentence in the previous caption. 


$$
\Delta=\lambda \int_{0}^{\omega D} d \varepsilon \tanh \left(\frac{\varepsilon}{2 T}\right) \operatorname{Re} \frac{1}{\sqrt{u^{2}-1}}
$$

and $\gamma=1 / \tau_{S} \Delta$ is the pair breaking parameter $\left(\tau_{S}\right.$ is the mean free time during, e.g., spin-flip scattering at impurities). When magnetic impurities are absent, $\tau_{S}$ tends to infinity and Eq. (1) coincides with the equation for current in Ref. 24. The energy gap $\Delta_{0}$ and the order parameter $\Delta$ are related as follows:

$$
\Delta_{0}=\Delta\left(1-\gamma^{2 / 3}\right)^{3 / 2} .
$$

The other approach was based on the generalized

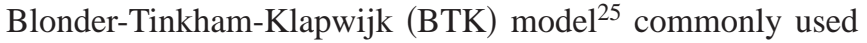
to describe $S-c-N$ point contacts. The model allows for the finite lifetime of quasiparticles $\tau=\hbar / \Gamma$ determined by inelastic scattering, which leads to the broadening of the density of states in the superconductor. Formally, according to the theory ${ }^{18}$ the BTK-based results are obtained under the condition of strong pair breaking $(|u| \gg 1)$. Therefore, in the strict sense, the generalized BTK model contains no gap. For any infinitesimal broadening parameter $\Gamma$, at $T=0$ the density of states near the Fermi surface is nonzero. In theory, ${ }^{18}$ the order parameter is a quantity analogous to the pseudogap in the generalized BTK model. In the following we use terms "order parameter" and $\gamma$ for the model of Ref. 18, while the "gap" and $\Gamma$ for the commonly used BTK model, ${ }^{25}$ except special cases, where we refer to $\Delta_{0}$ related to the model. ${ }^{18}$

Now we consider more closely the methods of fitting the theoretical curves to the experimental results. The iteration method commonly used in this case is quite good for small broadening. However, when the broadening $\Gamma$ is comparable with the gap (or $\gamma>0.3$ ), the results thus obtained are ambiguous and often dependent on the starting $\Delta, \Gamma$ (or $\gamma$ ) and $Z$ values. In our calculation, we therefore used the technique of coordinate descent with a postponed solution.

First, we specified an interval in which $\Delta$ is searched at a given temperature. The interval was then subdivided into equidistant parts, $\Delta_{1}, \Delta_{2}, \ldots, \Delta_{n} . \gamma$ and $Z$ were fitted for each $\Delta_{i}$. The procedure was as follows: after each step of $\gamma$-fitting, a complete $Z$-fitting was performed and then the next step of $\gamma$-fitting was considered. Before each calculation of the average rms deviation $F\left(\Delta_{i}\right)$, the amplitudes of the fitting and the experimental curves were made equal by multiplying the $y$-coordinate of the fitting curve by a scale-factor $S$ and then shifting it along the $y$-axis by an amount $B$. The values of $B$ and $S$ were chosen to minimize the deviation $F\left(\Delta_{i}\right)$. The standard algorithm for determination of $B$ and $S$ is considered, for instance, in Ref. 26. As a result, for each $\Delta_{i}$ we found $\gamma_{i}$ and $Z_{i}$ at which the difference between the shapes of the theoretical and experimental curves characterized by the rms deviation $F\left(\Delta_{i}\right)$ was the smallest one. The same holds for $\Gamma$ in the BTK fitting model. ${ }^{25}$ The calculation for some temperatures is shown in Fig. 3. It is evident that at $T_{c}$ the technique cannot ensure unambiguous results. At $14.5 \mathrm{~K}$, the curve $F\left(\Delta_{i}\right)$ is practically horizontal. The determination of the $\Delta$-value should take into account other factors as well. For example, it is important that the tunneling parameter $Z$ and the scale factor $S$ should be invariant. The

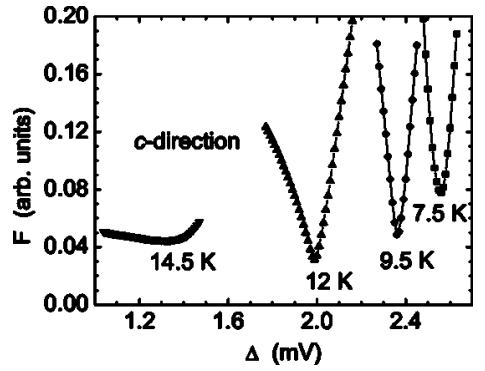

FIG. 3. Averaged rms deviation $F$ characterizing the extent of shape discrepancy between the theoretical and experimental curve as a function of $\Delta$. The minima in the curves correspond to the best agreement of theory and experiment. The data shown are for $\mathrm{LuNi}_{2} \mathrm{~B}_{2} \mathrm{C}$-Ag point contacts in the $c$ direction at different temperatures. Calculation based on Ref. 18.

dependences $S(\Delta)$ and $F(\Delta)$ at $T=7.5 \mathrm{~K}$ are shown in Fig. 4. It is seen that the minimum $F$ corresponds to a certain $S$ value. The BTK calculation of the corresponding dependences at the same temperature yields broader $F(\Delta)$ curves. Nevertheless, the minima positions in these curves are quite definite.

At low temperatures the shapes of the theoretical and the experimental curves were in rather poor agreement for all of the borocarbides investigated $(\mathrm{Re}=\mathrm{Er}, \mathrm{Dy}, \mathrm{Tm}, \mathrm{Lu})$ irrespective of the point contact orientation. The temperature dependence of the rms deviation in shape in the $c$ direction is shown in Fig. 5 for both models. ${ }^{18,25}$ It is seen that the best agreement is achieved above $7 \mathrm{~K}$. At $T<7 \mathrm{~K}$, the largest discrepancy between the shapes is observed in the region of extrema. The central maximum of the differential resistance in the experimental curve is considerably narrower than that in the best fit, ${ }^{29}$ whereas the minima are shifted to higher bias (Fig. 6).

Some experimental curves have a kink marked with an arrow in Fig. 6. This shape is typical of a superconductor with two gaps of close energies. The temperature dependence of the broadening (pair breaking) parameter also supports the existence of two gaps. Since Lu is a nonmagnetic material, it is natural to expect a negligible broadening (pair breaking) parameter for the Lu-based borocarbide point contacts. However, that is not the case, as is evident from the temperature dependences (Fig. 7) of the broadening (pair breaking) parameters obtained within the two models (Ref. 18 and $\left.\mathrm{BTK}^{25}\right)$.

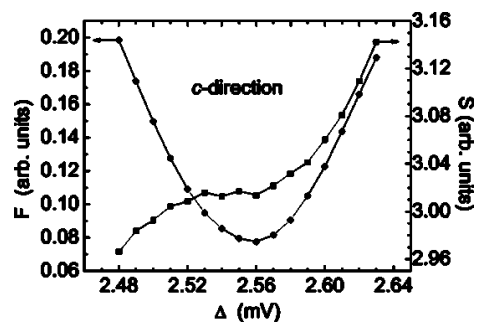

FIG. 4. $\Delta$-dependences of the averaged rms deviation $F$ (see Fig. 3) and the scale factor $S$ at $T=7.5 \mathrm{~K}$. Calculation by equations from Ref. 18. $S$ is a factor used to divide the $y$-coordinate of the theoretical curve to match its amplitude with that of the experimental curve. 


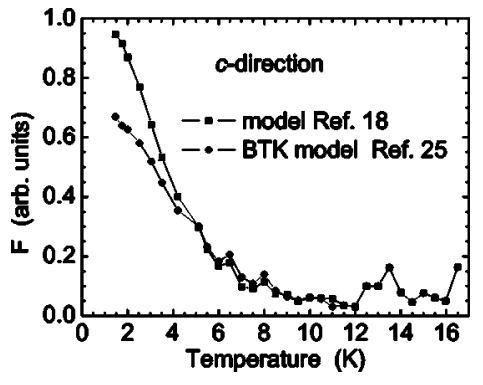

FIG. 5. Temperature dependence of the shape discrepancy between theoretical and experimental curves calculated according to Ref. 18 and within the generalized BTK model (Ref. 25) for $\mathrm{LuNi}_{2} \mathrm{~B}_{2} \mathrm{C}-\mathrm{Ag}$ point contacts in the $c$ direction.

Note that $\gamma$ and $\Gamma$ are different quantities. In the generalized BTK model ${ }^{25} \Gamma$ is independent of the gap and has the gap dimensions; whereas, in the model of Ref. 18, $\gamma$ is described as $\gamma=1 / \tau_{S} \Delta$, and, for $\gamma<1, \gamma$ is related to the gap $\Delta_{0}$ and the order parameter $\Delta$ as $\gamma=\left[1-\left(\Delta_{0} / \Delta\right)^{2 / 3}\right]^{3 / 2}$. As the temperature is increased, both $\Gamma$ and $\gamma$ decrease, and, at temperatures $>7.5 \mathrm{~K}$ for the $a b$-plane and $>11 \mathrm{~K}$ for the $c$-direction, tend to zero. Meanwhile, the one-gap fit starts to approximate the experimental curve more and more accurately. In this case the one gap fit with the broadening (pair breaking) parameter is a tool of describing a certain average gap (order parameter).

The largest and smallest gaps can be estimated by fitting different portions of the experimental curve. In this case, to match the amplitudes of the fitting and the experimental curves, we used another method. Namely, the $y$-coordinates at the central maximum and near the minima are scaled to coincide (Fig. 8). The best agreement in the central maximum region can give us an estimate of the smallest gap (curve 1), while the best agreement in the "wing" region yields the largest gap (curve 2). By "wings" we imply the portion of the experimental curve at biases higher than the energy gap double-minimum structure in the $d V / d I$ characteristic, namely at $e V \gtrsim 3 \mathrm{meV}$. The estimates however are rather rough.

Further, we follow the method of approximation of $I-V$ characteristics, which was calculated for the $S-I-N$ contacts of $\mathrm{MgB}_{2}$ within a two-band model in Ref. 17. There, the

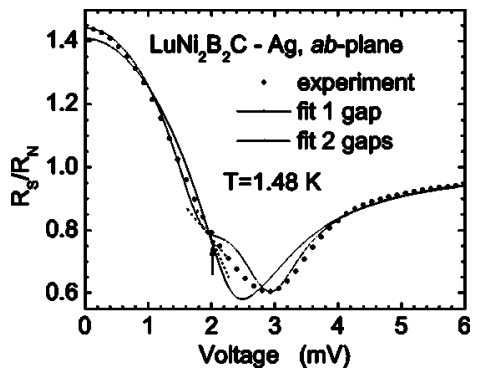

FIG. 6. Approximation of the experimental curve with one- and two-gap models for the $\mathrm{LuNi}_{2} \mathrm{~B}_{2} \mathrm{C}-\mathrm{Ag}$ point contact in the $a b$ plane. The crossing straight lines marked with an arrow indicate the kink in the experimental curve which is exaggerated by the two-gap theoretical fit.

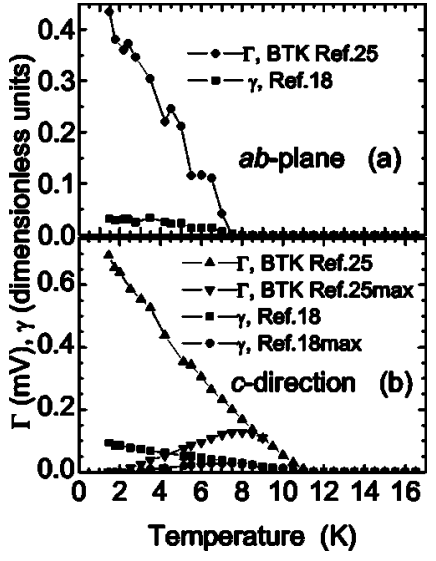

FIG. 7. Temperature dependences of the broadening and pair breaking parameters for the $\mathrm{LuNi}_{2} \mathrm{~B}_{2} \mathrm{C}-\mathrm{Ag}$ point contacts in the $a b$ plane (a), and along the $c$ axis (b). The index "max" marks the "wings" fitting (see text). "Wings" fit for $a b$-plane gives $\gamma=0$.

total conductivity of the tunnel contact is a sum of the $\pi$ - and $\sigma$ - band conductivities analyzed by applying the BTK model. To describe the resulting curve, we also used a model of two independent parallel-connected point contacts with different gaps whose conductivities are additive. The contributions of these conductivities account for the part of the Fermi surface containing a particular gap. Thus, for the two-gap model an experimental curve is fitted by the following expression:

$$
\frac{d V}{d I}=\frac{S}{\frac{d I}{d V}\left(\Delta_{1}, \gamma_{1}, Z\right) K+\frac{d I}{d V}\left(\Delta_{2}, \gamma_{2}, Z\right)(1-K)},
$$

with a proper choice of the coefficient $B$. Here, the coefficient $K$ reflects the contribution of the part of the Fermi surface having the gap $\Delta_{1}, S$ is the scaling factor discussed for the one-gap approximation. To obtain the best agreement with the experiment, the parameters used in this expression are allowed to differ from those found for fitting of the separate portions of the experimental curve. It appears that when

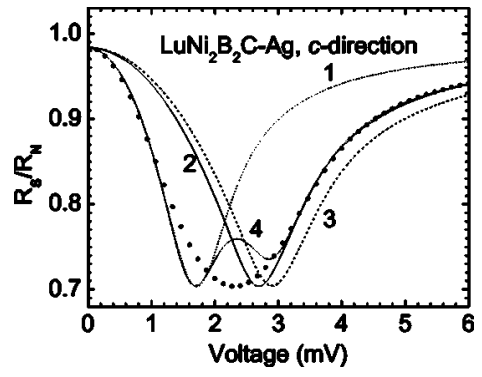

FIG. 8. The estimates of the smallest and largest gaps for the $\mathrm{LuNi}_{2} \mathrm{~B}_{2} \mathrm{C}-\mathrm{Ag}$ point contact in the $c$ direction at $T=1.47 \mathrm{~K}$. Curve $1, \Delta=1.82 \mathrm{meV}\left(\Delta_{0}=1.65 \mathrm{meV}\right), \gamma=0.016, Z=0.558$; curve $2, \Delta$ $=2.97 \mathrm{meV}\left(\Delta_{0}=2.693 \mathrm{meV}\right), \gamma=0.016, Z=0.553$; curve $3, \Delta$ $=3.2 \mathrm{meV}\left(\Delta_{0}=2.9 \mathrm{meV}\right), \gamma=0.016, Z=0.558$. The best two-gap approximation (curve 4 ) is achieved with the parameters of curves 1 and 3 . The relative contribution to the total conductivity is $60 \%$ from the smaller gap and $40 \%$ from the larger gap (see Table I). The experimental curve is shown with solid dots. 
TABLE I. The fitting results for superconducting parameters along two mutually perpendicular directions, using Ref. 18 and the BTK theory (Ref. 25). $\Delta_{1}, \Delta_{2}$ are the order parameters in the two-gap fitting; whereas, the subindex 0 corresponds to the energy gaps in the model of Ref. 18. The pair breaking parameters $\gamma_{1,2}$ and the broadening parameter $\Gamma_{1,2}$ correspond to Refs. 18 and 25 , respectively. $Z$ is the tunneling parameter. The temperature corresponds to that of the experiment. The relative contributions of each superconducting order parameter and gap are given in percent. $\Delta$ and $\Gamma$ are given in $\mathrm{meV}, \gamma$ and $Z$ are dimensionless.

\begin{tabular}{|c|c|c|c|c|c|c|c|c|c|c|c|c|}
\hline \multicolumn{8}{|c|}{ Two-gap fit } & & \multicolumn{4}{|c|}{ One-gap fit } \\
\hline & \multicolumn{3}{|c|}{ Reference 18} & & \multicolumn{3}{|c|}{ BTK (Ref. 25) } & & \multicolumn{2}{|c|}{ Reference 18} & \multicolumn{2}{|c|}{ BTK (Ref. 25) } \\
\hline & $c$ & & $a b$ & & $c$ & & $a b$ & & $c$ & $a b$ & $c$ & $a b$ \\
\hline \multirow[t]{2}{*}{$\Delta_{1}$} & 3.2 & & 3.03 & & & & & & 2.66 & 2.74 & & \\
\hline & & $40 \%$ & & $60 \%$ & 2.65 & $50 \%$ & 3 & $62 \%$ & & & 2.25 & 2.55 \\
\hline$\Delta_{01}$ & 2.9 & & 2.92 & & & & & & 1.88 & 2.34 & & \\
\hline \multirow[t]{2}{*}{$\Delta_{2}$} & 1.82 & & 2 & & & & & & & & & \\
\hline & & $60 \%$ & & $40 \%$ & 1.7 & $50 \%$ & 2.2 & $38 \%$ & & & & \\
\hline$\Delta_{02}$ & 1.65 & & 1.82 & & & & & & & & & \\
\hline \multirow[t]{2}{*}{$\gamma_{1}, \Gamma_{1}$} & 0.016 & & 0.004 & & 0.4 & & 0.1 & & & & & \\
\hline & & & & & & & & & 0.094 & 0.031 & 0.655 & 0.434 \\
\hline$\gamma_{2}, \Gamma_{2}$ & 0.016 & & 0.012 & & 0.4 & & 0.14 & & & & & \\
\hline$Z$ & 0.558 & & 0.765 & & 0.59 & & 0.77 & & 0.55 & 0.745 & 0.6 & 0.804 \\
\hline$T(\mathrm{~K})$ & 1.47 & & 1.48 & & 1.47 & & 1.48 & & 1.47 & 1.48 & 1.47 & 1.48 \\
\hline
\end{tabular}

the contribution of the smaller gap prevails, the "wings" fit gives smaller values. In this case, the best result is achieved with the two-gap fit (Fig. 8, curve 4) using the parameters for curves 1 and 3 , the latter is marked with dots. Correspondingly, if the larger gap prevails, the central-maximum fit gives higher values. In Fig. 6 (dotted curve) an example of the two-gap fit for the $a b$-plane is shown.

Although the two-gap fit shows much better agreement in the regions both of the central maximum and the "wings" (see Figs. 6 and 8), it cannot provide a complete description of the experimental curves, especially at the double-minima structure of $d V / d I$, and in the $c$ direction (Fig. 8). Most likely, this is because of a continuous distribution of the gaps over the Fermi surface.

Our estimation of point-contact parameters in the $a b$ plane and in the $c$ direction was made within one-gap and two-gap models using the equations of Ref. 18 and BTK. ${ }^{25}$ The results are presented in Table I. $\Delta_{1}, \Delta_{2}$ are the highest and lowest order parameters (the same as BTK gaps ${ }^{25}$ ) from the two-gap fit; $\Delta_{01}$ and $\Delta_{02}$ are the energy gaps corresponding to $\Delta_{1}, \Delta_{2} ; \gamma_{1}, \gamma_{2}$ are the pair breaking parameters of the model $^{18}$ for the largest and smallest gaps. The same holds for the widening parameters $\Gamma_{1}, \Gamma_{2}$ in the BTK model. ${ }^{25}$ Independently determined Z's are the tunneling parameters, which are the same for the largest and smallest gaps and are thus quite self-consistent. In Table I the order parameters (gaps) based on the two-gap fit appear along with the contributions to the total conductivity from the Fermi surface region with the corresponding gap. For example, for the $c$ direction contact, the contribution to the conductivity is $40 \%$ from the region with an order parameter of $3.2 \mathrm{mV}$ and $60 \%$ from the region with $1.82 \mathrm{mV}$.

It is seen that within both the models, ${ }^{18,25}$ the dominant contribution to the total conductivity in the $c$ direction is made by the Fermi surface region where the order parameter is lower. The higher order parameter makes the dominant contribution in the $a b$ plane. On the average this correlates with gaps in $a b$ and $c$ directions predicted by the $(s+g)$ model. ${ }^{11}$

Although the part-by-part fitting is rather rough, we tried this procedure for tracing the temperature dependences of the smallest and largest gaps. However, more or less definite values were obtained only at the lowest temperatures because the smallest gap is estimated within a relatively small part of the experimental curve (near zero bias). As for the largest gap, its temperature dependence was traced up to the moment when the calculations over the entire curve and over the "wings" start to give similar results.

The temperature dependences of the scale factor $S$ in the $c$ direction are shown in Fig. 9. They were found for the largest gap ("wing" fitting is marked with "max" throughout) and for the average gap (entire-curve fitting) by the equations of Ref. 18 and BTK. ${ }^{25}$ The temperature dependences of the tunneling parameter $Z$ are shown for both models. To avoid crowding in the figure, only the $Z$-values obtained from the

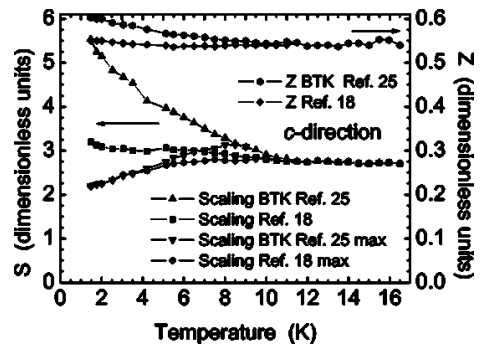

FIG. 9. Temperature dependences of the tunneling parameter $Z$ and the scale factor $S$ for the $\mathrm{LuNi}_{2} \mathrm{~B}_{2} \mathrm{C}-\mathrm{Ag}$ point contact in the $c$ direction obtained with models from Refs. 18 and 25 . The scale factor was found from fitting over the entire curve and over the "wings" (scaling max). 


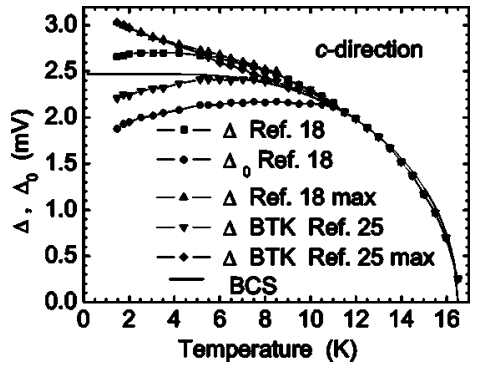

FIG. 10. Temperature dependence of the order parameter $\Delta$ and energy gap $\Delta_{0}$ for the one-gap fitting models (Refs. 18 and 25) in the $c$ direction. The $T$-dependence of the largest order parameter, indicated by max, was estimated from fitting over the "wings."

entire-curve fitting are shown (the "wing"-fitting results are much the same). Although the fitting was focused on the coincidence of theoretical and experimental $d V / d I(V)$ curves and no parameters were specially fixed, we can state that $Z$ vary very little in the whole temperature interval as one could expect. The divergence of $S(T)$ curves at low temperatures depends upon the model pointing that none of them correctly accounts for the gap anisotropy. At $T \geqslant 11 \mathrm{~K}$ the "wing" and entire-curve fits start to yield similar results.

The temperature dependences of the order parameter and the gap in the $c$ direction, obtained both by the entire-curve and the "wing" one-gap fittings, ${ }^{18,25}$ are shown in Fig. 10 along with the BCS curve. As in the case of the scaling factor $\mathrm{S}$ (Fig. 9), deviation starts at $T \lesssim 11 \mathrm{~K}$, while at higher temperature all the fitting procedures result in the same BCS-like dependence.

The temperature dependences of the order parameter ${ }^{18,25}$ and the gap ${ }^{18}$ for the $a b$ plane contact are shown in Fig. 11. The calculation was made both over the entire curve and the "wings" as in Fig. 10 by means of one-gap fitting according to Refs. 18 and 25. It is seen that the curves coincide in a wider temperature interval compared to the $c$ direction and follow the BCS-dependence down to about $8 \mathrm{~K}$. Thus, the gaps in the $a b$ plane and $c$ direction differ not only in magnitude but in temperature dependence as well. In the $a b$ plane different one-gap fitting models lie closer to the BCS standard curve compared to those in the $c$ direction. We in-

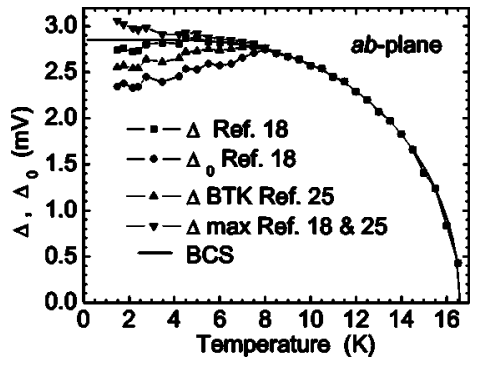

FIG. 11. Temperature dependence of the order parameter $\Delta$ and energy gap $\Delta_{0}$ (calculation by Ref. 18) and the BTK energy gap (calculation by Ref. 25) for the one-gap fitting models in the $a b$ plane. The $T$-dependence of the largest gap (order parameter) was estimated from fitting over the "wings." The scatter of the points on the same curve at $T=1.5-4.2 \mathrm{~K}$ is caused by the contact instability. The contact resistance becomes stable above $4.5 \mathrm{~K}$. terpret this feature that in the $a b$ direction the point-contact average gap anisotropy is less than in the $c$ orientation. One can see that the "wings" fitting $\Delta(T)$ goes above BCS lowtemperature limit while the entire-curve fitting falls below the BCS standard dependence. We emphasize that the difference between the BCS and experimental dependencies is due to a poor fitting by any of the one-gap models at low temperatures.

Let us consider in more detail the recent investigation of the superconducting gap anisotropy in Lu and Y compounds by means of STM. ${ }^{19}$ The tunneling spectra obtained in this work appeared to be impossible to fit in terms of the traditional BCS theory. For the curve shown in Fig. 2 (Ref. 19), the zero value of the differential conductivity is observed only below $0.8 \mathrm{mV}$ and the maximum of $d I / d V$, which corresponds approximately to the energy gap, is located at $2.3 \mathrm{meV}$. At the same time, the shape of the curve is permanent along the surface area much greater than the coherence length. In Fig. 2(b), at energies slightly less than $2 \mathrm{meV}$, there is a shoulder, which could be ascribed to a two-gap spectrum. Unfortunately, this feature is not discussed in Ref. 19. The authors also notice that the use of the model, where the broadening of the BCS density of states is caused by the finite lifetime of quasiparticles, implies a nonzero density of states (or differential conductivity) at infinitesimal bias which is at odds with the experimental result. Because of that, the authors of Ref. 19 use a modified density of state modeled by the energy gap with the Gauss distribution centered at $\Delta$ with the width $\epsilon$. By investigation of different parts of the crystal surface, the authors of Ref. 19 observe the correlation between the supposed anisotropy of the gap expressed in $\epsilon / \Delta$ units and the local transition (critical) temperature at the given spot on the crystal surface. With increase of the critical temperature under the contact, both the absolute value of the gap and its anisotropy increase. Such a behavior corresponds to the $s$-wave pairing, since in terms of that model the elastic scattering leads to a decrease of $T_{c}$ and isotropy of the gap.

In our experiment the critical temperature coincides for both directions with the bulk value, as one could expect in a crystal with undisturbed surface. Our parameter $\Gamma$ allows one to estimate quantitatively the gap anisotropy and is similar to the parameter $\epsilon / \Delta$ in Ref. 19. Namely, $\Gamma$ is about 1.5 times greater in the $c$ direction compared with that in the $a b$ plane. The temperature interval where our gap dependences deviate from BCS curve is also about 1.5 times larger in the $c$ direction compared with that in the $a b$ plane. Hence, we may conclude that the anisotropy of the superconducting gap in the $c$ direction is greater than in the $a b$ plane.

Note that neither of the techniques ${ }^{18,25}$ can describe adequately the presumed situation because in both cases the discrepancy is determined by the distribution of the gap over the Fermi surface. Taking into account the poor efficiency of the one-gap fit at low temperatures (Fig. 5) and the deviation of the fitting curve at the minima of $d V / d I$ for the two-gap fit (see Figs. 6 and 8 which give approximately the same discrepancy factor $F$ as for the lowest temperature in Fig. 5), we can assert that the superconducting gap varies continuously over the Fermi surface. It is therefore most reasonable to describe such curves in terms of a number of parallel- 


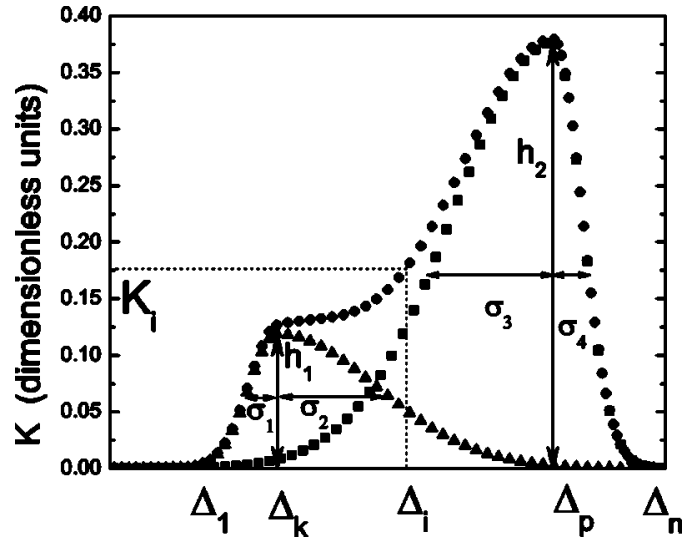

FIG. 12. Modeling of the anisotropic superconducting energy gap distribution over the Fermi surface with multiple parallel junctions of different gaps. The contribution of a particular energy gap correlates with its part over the Fermi surface. The distribution of a particular contribution to the total conductivity is described by the $K_{i}\left(\Delta_{i}\right)$ dependence. The shape of the distribution $K_{i}\left(\Delta_{i}\right)$ is simulated by superposition of two peaks. Each peak is described by the following parameters: the energy of the central maximum $\Delta_{k}$ or $\Delta_{p}$, half-width of the right and left slopes $\sigma_{1}, \sigma_{2}$ or $\sigma_{3}, \sigma_{4}$, and the heights $h_{1}$ and $h_{2}$, correspondingly. By variation of these eight parameters we attempt to reach the best coincidence between the experimental and theoretical curves.

connected point contacts having different gaps. Their contribution to the total conductivity can qualitatively account for the region of the Fermi surface containing a particular gap, as was considered in Ref. 27.

Let us illustrate this approach by fitting the experimental curves for the $c$ - and $a b$-directions at the lowest temperatures, where the discrepancy is the highest (Figs. 6 and 8). Just as the two-gap fit (5), the multigap fit is expressed by

$$
\frac{d V}{d I}=\frac{S}{\frac{d I}{d V}\left(\Delta_{1}, Z\right) K_{1}+\cdots+\frac{d I}{d V}\left(\Delta_{n}, Z\right) K_{n}},
$$

where $K_{1}+K_{2}+\cdots+K_{n}=1, K_{i}$ is proportional to the part of the Fermi surface with the gap $\Delta_{i}$, and $S$ is the scaling factor. The broadening parameter $(\gamma$ or $\Gamma)$ was taken to be zero, and the barrier factor $Z$ was the same for all the contributions. In this approximation the approaches of Refs. 18 and 25 are the same, and the order parameter coincides with the energy gap. As a first approximation, it is supposed that the coefficients $K_{i}$ are located in the curve which is a superposition of two peaks (Fig. 12). The maxima of these peaks are at energies $\Delta_{k}$ and $\Delta_{p}$, and their slopes are described by asymmetrical Gaussian distributions with a width at half-height of $\sigma_{1}, \sigma_{2}$, $\sigma_{3}$, and $\sigma_{4}$. The heights of each peak are $h_{1}$ and $h_{2}$, respectively. By varying all the fitting parameters $\left(\Delta_{i}, h_{i}, \sigma_{k}\right.$ for $i$ $=1,2$ and $k=1-4$ ) we minimized the average rms deviation. This procedure includes manually offsetting dots of the $K(\Delta)$ fitting curve in the intermediate steps of the adjustment.

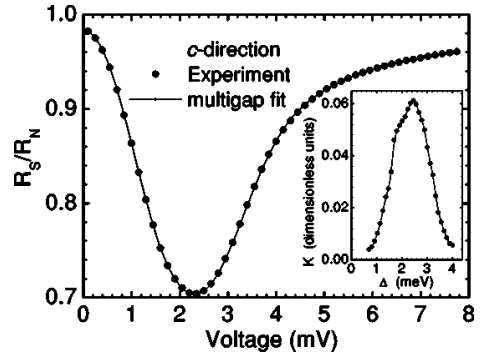

FIG. 13. Approximation of the experimental curve by the use of the multigap model for the $c$ direction. $T=1.47 \mathrm{~K}, Z=0.559$. Inset, the contribution to the total conductance from different parts of the Fermi surface with different gaps. The distribution is modeled by 31 equidistant points, $\Delta_{\min }=0.7 \mathrm{meV}, \Delta_{\max }=4 \mathrm{meV}$. The resulting curve exibits two peaks with maxima at $1.8-2 \mathrm{meV}$ and $2.5 \mathrm{meV}$.

The result is shown in Figs. 13 and 14 for the $c$ - and $a b$-directions, respectively. One can see that the multigap fit with eight fitting parameters and a continuous distribution of the gaps matches the experimental curve very well. One should not trust this fitting literally, but it shows that the width of the gap distribution can be quite appreciable, down to small values, which agrees with the tunneling results ${ }^{19}$ and theoretical $(s+g)$ model of the gap nodes in [100] directions. ${ }^{11}$

\section{CONCLUSION}

In this study the anisotropy of the superconducting energy gap was measured for the first time on $\mathrm{LuNi}_{2} \mathrm{~B}_{2} \mathrm{C}$ in the $a b$ plane and $c$ directions. It is found that at low temperatures the experimental curves should be described assuming a gap distribution over the Fermi surface. Within the two-gap model, the largest contribution to the total conductivity is made by the Fermi region with a smaller gap in the $c$ direction and by the Fermi region with a greater gap in the $a b$ plane. At $T \simeq 1.5 \mathrm{~K}$ the largest and the smallest order parameters in the $c$ direction are $\Delta_{\max }=3.2 \mathrm{meV}, \Delta_{\min }=1.82 \mathrm{meV}$; in the $a b$ plane these are $\Delta_{\max }=3.03 \mathrm{meV}, \Delta_{\min }=2.0 \mathrm{meV}$. An attempt to fit the low temperature experimental curves

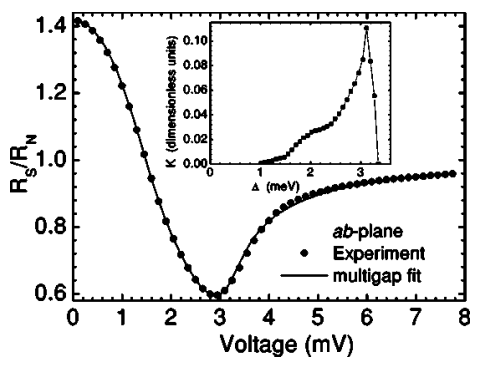

FIG. 14. Approximation of the experimental curve by the use of the multigap model for the $a b$ plane. $T=1.48 \mathrm{~K}, Z=0.755$. Inset, the contribution to the total conductance from different parts of Fermi surface with different gaps. The distribution is modeled by 31 equidistant points, $\Delta_{\min }=1.0 \mathrm{meV}, \Delta_{\max }=3.35 \mathrm{meV}$. The resulting curve exibits two peaks with maxima at $2 \mathrm{meV}$ and $3.1 \mathrm{meV}$. 
shows that the gap is distributed starting from $0.8 \mathrm{meV}$. This corresponds well to the recent STM observations in Ref. 19 discussed above. We have found the deviation of the temperature dependent gap from the BCS theory for both tested directions. This deviation is connected with the impossibility to describe the gap distribution in terms of the one-gap model, at least at low temperatures. The temperature range where the described deviation is observed for the $c$ direction, is about 1.5 times greater $(1.5-11.5 \mathrm{~K})$ than that in the $a b$ plane $(1.5-7.5 \mathrm{~K})$. The broadening parameter $\Gamma$, allowing quantitative estimation of the degree of anisotropy, is also bigger in the $c$ direction.

The experimental results are described on the basis of the generalized BTK model ${ }^{25}$ and the Beloborod'ko theory, ${ }^{18}$ considering the electrical conductivity of ballistic $S-c-N$ point contacts in the presence of an arbitrarily penetratable potential barrier and allowing for the finite lifetime of Cooper pairs. For superconductors with a multiband electronic structure, the interband transitions of Cooper pairs may lead to their finite lifetime. Previously, a two-band model was suggested for nickel borocarbide superconductors. ${ }^{28}$ The theory, ${ }^{18}$ which accounts more accurately for the force of pair breaking, may explain the difference between the order parameter and the gap.

Our next publication will concern the compound $\mathrm{ErNi}_{2} \mathrm{~B}_{2} \mathrm{C}$ which exhibits a magnetic transition near $T=6 \mathrm{~K}$. In this context a detailed analysis and comparison of the two theoretical approaches ${ }^{18,25}$ are of paramount importance for understanding the results that can be obtained on magnetic $\mathrm{ErNi}_{2} \mathrm{~B}_{2} \mathrm{C}$.

\section{ACKNOWLEDGMENTS}

The single crystal samples for this study were graciously provided by P. C. Canfield and S. L. Bud'ko at Ames Laboratory and Iowa State University. Much of the work reported here was supported in part by the Robert A. Welch Foundation (Grant No. A-0514), the Telecommunications and Informatics Task Force at Texas A\&M University, the Texas Center for Superconductivity and Advanced Materials at the University of Houston (TCSAM), and the National Science Foundation (Grant Nos. DMR-0103455 and DMR-0111682). Partial support of U.S. Civilian Research and Development Foundation for the Independent States of the Former Soviet Union (Contract No. UP1-2566-KH-03) is acknowledged.
*Email address: yanson@ilt.kharkov.ua

${ }^{1}$ L. M. Volkova, S. A. Polyshchuk, S. A. Magarill, and F. E. Herbeck, cond-mat/0312540 (unpublished).

${ }^{2}$ K-H. Muller and V. N. Narozhnyi, Rep. Prog. Phys. 64, 943 (2001).

${ }^{3}$ K. D. D. Rathnayaka, A. K. Bhatnagar, A. Parasiris, D. G. Naugle, P. C. Canfield, and B. K. Cho, Phys. Rev. B 55, 8506 (1997).

${ }^{4} \mathrm{~K}-\mathrm{H}$. Muller et al., in Handbook of Magnetic Materials, edited by K. H. J. Buschow (Elsevier Science, New York, 2002), Vol. 14, pp. 199-305.

${ }^{5}$ V. Metlushko, U. Welp, A. Koshelev, I. Aronson, G. W. Crabtree, and P. C. Canfield, Phys. Rev. Lett. 79, 1738 (1997).

${ }^{6}$ E. Boaknin, R. W. Hill, C. Proust, C. Lupien, L. Taillefer, and P. C. Canfield, Phys. Rev. Lett. 87, 237001 (2001)

${ }^{7}$ K. Izawa, K. Kamata, Y. Nakajima, Y. Matsuda, T. Watanabe, M. Nohara, H. Takagi, P. Thalmeier, and K. Maki, Phys. Rev. Lett. 89, 137006 (2002).

${ }^{8}$ T. Park, M. B. Salamon, E. M. Choi, H. J. Kim, and S.-I. Lee, Phys. Rev. Lett. 90, 177001 (2003).

${ }^{9}$ T. Watanabe, M. Nohara, T. Hanaguri, and H. Takagi, Phys. Rev. Lett. 92, 147002 (2004).

${ }^{10}$ K. Maki, P. Thalmeier, and H. Won, Phys. Rev. B 65, 140502(R) (2002)

${ }^{11} \mathrm{P}$. Thalmeier and G. Zwicknagl, cond-mat/0312540 (unpublished).

${ }^{12}$ G. E. Blonder, M. Tinkham, and T. M. Klapwijk, Phys. Rev. B 25, 4515 (1982).

${ }^{13}$ Y. De Wilde, M. Iavarone, U. Welp, V. Metlushko, A. E. Koshelev, I. Aranson, G. W. Crabtree, and P. C. Canfield, Phys. Rev. Lett. 78, 4273 (1997).

${ }^{14}$ Y. De Wilde, M. Iavarone, U. Welp, V. Metlushko, A. E. Ko- shelev, I. Aranson, G. W. Crabtree, P. L. Gammel, D. J. Bishop, and P. C. Canfield, Physica C 282-287, 355 (1997).

${ }^{15}$ I. K. Yanson, N. L. Bobrov, C. V. Tomy, and D. McK. Paul, Physica C 334, 33 (2000).

${ }^{16}$ L. F. Rybalchenko, I. K. Yanson, A. G. M. Jansen, P. Mandal, P. Wyder, C. V. Tomy, and D. McK. Paul, Physica B 218, 189 (1996).

${ }^{17}$ A. Brinkman, A. A. Golubov, H. Rogalla, O. V. Dolgov, J. Kortus, Y. Kong, O. Jepsen, and O. K. Andersen, Phys. Rev. B 65, 180517 (2002).

${ }^{18}$ S. I. Beloborod'ko, Fiz. Nizk. Temp. 29, 868 (2003) [Low Temp. Phys. 29, 650 (2003)].

${ }^{19}$ P. Martinez-Samper, H. Suderow, S. Vieira, J. P. Brison, N. Luchier, P. Lejay, and P. C. Canfield, Phys. Rev. B 67, 014526 (2003).

${ }^{20}$ B. K. Cho, P. C. Canfield, and D. C. Johnston, Phys. Rev. B 52, R3844 (1995).

${ }^{21}$ P. N. Chubov, A. I. Akimenko, and I. K. Yanson, Author Certificate: Patent No. 83408 (USSR); A Method for Obtaining Pressed Microcontacts Between Metallic Electrodes (Bulletin Izobretenii, Moscow, 1981), No. 20.

${ }^{22}$ B. N. Engel, G. G. Ihas, E. D. Adams, and C. Fombarlet, Rev. Sci. Instrum. 55, 1489 (1984).

${ }^{23}$ A. A. Abrikosov and L. P. Gorkov, Zh. Eksp. Teor. Fiz. 39, 1781 (1960) [Sov. Phys. JETP 12, 1243 (1961)].

${ }^{24}$ A. V. Zaitsev, Zh. Eksp. Teor. Fiz. 86, 1742 (1984) [Sov. Phys. JETP 59, 1015 (1984)].

${ }^{25}$ A. Plecenik, M. Grajcar, S. Benacka, P. Seidel, and A. Pfuch, Phys. Rev. B 49, 10016 (1994).

${ }^{26}$ D. Kahaner, C. Moler, and S. Nash, Numerical Methods and Software, 1st ed. (Prentice-Hall, Pearson Education Company, Upper Saddle River, NJ, 1989). 
${ }^{27}$ P. Martinez-Samper, J. G. Rodrigo, G. Rubio-Bollinger, H. Suderow, S. Vieira, S. Lee, and S. Tajima, Physica C 385, 233 (2003).

${ }^{28}$ S. V. Shulga, S.-L. Drechsler, G. Fuchs, K.-H. Müller, K. Winzer,
M. Heinecke, and K. Krug, Phys. Rev. Lett. 80, 1730 (1998).

29 "fit" stands for the theoretical $d V / d I(V)$ - dependence fitted to the experimental results. 